\title{
Extensive Regeneration in vitro by Early Embryonic Neurons on Immature and Adult CNS Tissue
}

\author{
Derryck Shewan, Martin Berry, and James Cohen \\ Division of Anatomy and Cell Biology, UMDS, Guy's Campus, London SE1 9RT, UK
}

The failure of axon regeneration in the injured adult CNS has been ascribed to axon growth inhibitory molecules expressed by the resident glial cell populations, especially oligodendrocytes. Unlike their adult counterparts, however, early embryonic neurons are able to send lengthy axons through myelinated fiber tracts when transplanted into the adult brain. One explanation is that they have yet to express receptors for factors that inhibit the growth of older neurons. To test this possibility, we have used the cryoculture technique to study the regeneration of rat central and peripheral neurons, over a developmental period that encompasses the stages before, during, and after target contact, when cultured on either unmyelinated (neonatal) or myelinated (adult) optic nerve tissue sections. Early embryonic (days 14-15) retinal ganglion cells extended neurites on neonatal optic nerve, but few grew on adult optic nerve. In the case of early embryonic dorsal root ganglion neurons, however, neurite outgrowth on elther neonatal optic nerve or on adult optic nerve was extensive. This response declined sharply with age. In contrast, neurite outgrowth by dorsal root ganglion neurons on laminin substrata remained relatively constant ( $>80 \%$ extended neurites) over the same period. This suggests that (a) inhibition of neurite outgrowth within the optic nerve is mediated not only by oligodendrocytes, but also by molecules expressed prior to the onset of myelination; (b) neurons acquire receptors for these inhibitors only late in embryonic development; (c) differences exist between developing central and peripheral neurons in the response to myelin-associated axon-growth inhibitors.

[Key words: axon regeneration, culture, DRG neurons, retinal ganglion cells, myelin, astrocytes, rat CNS]

The extent of regeneration in the injured adult mammalian nervous system appears to be primarily determined by the nature of the glial environment. Thus, the striking findings of Aguayo and colleagues (David and Aguayo, 1981; Benfey and Aguayo, 1982), that pieces of sciatic nerve grafted into the adult brain and spinal cord support long distance axon regeneration from juxtaposed injured neurons, suggest that Schwann cells, the glial cells of the PNS, combined with the abundant extracellular matrix in peripheral nerves, provide a favorable environment that

\footnotetext{
Received May 25, 1994; revised Aug. 22, 1994; accepted Sept. 7, 1994.

We thank Kevin Fitzpatrick and Sarah Smith for their assistance with photography. This work was supported by the International Spinal Research Trust and the Wellcome Trust. D.S. is the recipient of an Anatomical Society of Great Britain and Ireland research studentship.

Correspondence should be addressed to James Cohen at the above address. Copyright (c) 1995 Society for Neuroscience $0270-6474 / 95 / 152057-06 \$ 05.00 / 0$
}

fosters regeneration after injury in the PNS and, when transplanted, can do so in the injured CNS. In contrast, the resident glial cells in the adult CNS, oligodendrocytes and astrocytes, express molecules in the immediate vicinity of damaged axons that create an inhibitory environment for regenerating axons (reviewed in Johnson, 1994). However, the ability of CNS neurons to regenerate, even in the presence of sciatic nerve grafts, varies over a wide range (Fawcett, 1992). Moreover, as neurons age, new constraints are likely to be imposed on the regenerative response, due to changes, some possibly irreversible, in neuronal phenotype that accompany maturation (Fawcett et al., 1989; Bedi et al., 1992). Thus, reports of extensive axon regeneration by early human fetal forebrain neuroblasts following transplantation into the mature rat CNS (Wictorin et al., 1990, 1992) have focussed attention on possible intrinsic properties of immature neurons that enable them to extend processes in an environment that is inhospitable to axons regenerating from adult neurons. The underlying molecular bases for these differences are unknown.

Studying the mechanisms of axon regeneration in vivo, however, is impeded by the confusing variety of cell types, cell interactions, and trophic factors that may influence the behavior of the injured nerve fibers. Moreover, such experiments are complicated by accompanying degenerative changes. Conversely, in previous in vitro studies, insufficient account has been taken of the age of cultured neurons or of the complex environment encountered in vivo by regenerating neurites. To circumvent these problems, we have adopted the cryoculture assay system (Carbonetto et al., 1987; Covault et al., 1987; Sandrock and Matthew, 1987), which allows the study of neurite regeneration by dissociated neurons in vitro over intact, physiologically relevant substrata. Although the cryoculture method, being based on a two-dimensional assay environment, is only partially representative of the complex environment encountered by developing/regenerating neurons in vivo, it has the advantage of retaining extracellular matrix and cell surface molecules, while the influence of diffusible factors is minimized. In previous work using this technique, we have shown that neither neonatal and adult DRG neurons nor immature retinal ganglion cells (RGC) extend neurites when cultured on both immature, unmyelinated, and mature, myelinated CNS tissue sections (Shewan et al., 1993). In the present study, we have extended this approach, using the optic nerve as a prototype CNS tissue culture substratum, to examine the behavior of early embryonic neurons. Our data suggest that developing neurons acquire receptors for putative CNS growh-inhibitory molecules, only at late embryonic age. 

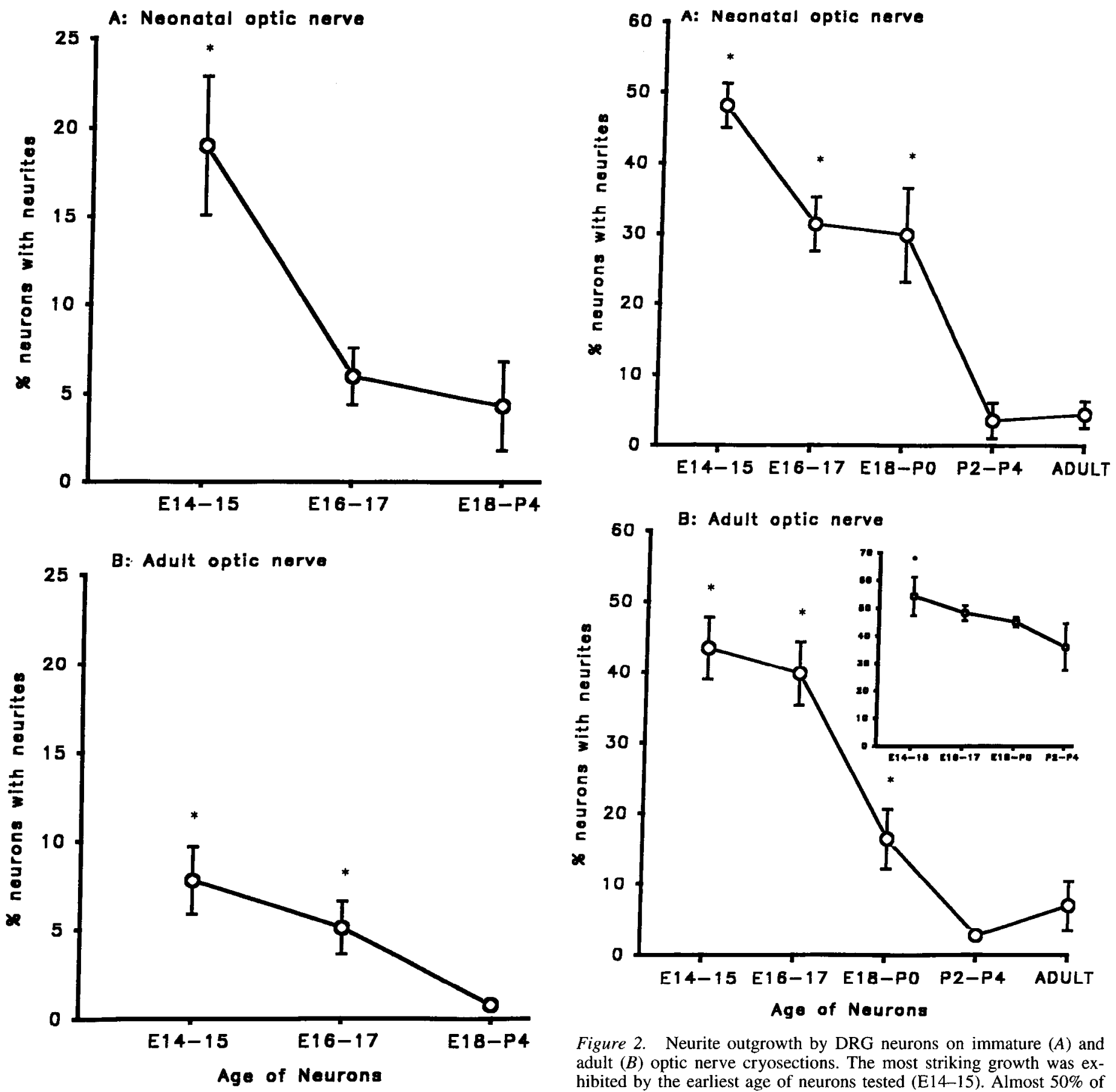

Figure 2. Neurite outgrowth by DRG neurons on immature $(A)$ and adult $(B)$ optic nerve cryosections. The most striking growth was exhibited by the earliest age of neurons tested (E14-15). Almost $50 \%$ of attached neurons of this age regenerated long neurites over both types of substratum. Note the similar developmental trend in declining outgrowth displayed on both the immature and mature optic nerve. Details as in legend to Figure 1, except that asterisks indicate values for age groups significantly different from $\mathrm{P} 2-4$ neurons $(p<0.05)$. Inset, Neurite outgrowth by DRG neurons on polylysine-coated glass over the age range depicted in Figure 2. Details as above.

Figure 1. Neurite outgrowth by RGC on immature $(A)$ and adult $(B)$ optic nerve cryosections over a developmental range encompassing the period during which RGC axons make target contact in vivo. The percentage of attached RGC extending long GAP43-positive neurites is greatest at the earliest embryonic ages tested (E13-15) on both substrata and progressively declines with maturation. Mean values plotted represent numbers of neurons with GAP43-positive neurites $\geq 3$ cbds \pm SEM of at least five separate experiments, statistically evaluated by the Student's two-tailed $t$ test. Asterisks indicate values for age groups significantly different from E18-P4 neurons $(p<0.05)$.

\section{Materials and Methods}

Tissue sections. Neonatal [embryonic day (E) 20 to postnatal day (P) 3], and adult optic nerves from Wistar rats, were carefully mounted longitudinally on pieces of liver mounted in embedding mediun (OCT compound; Tissue-Tek), taking care to exclude the OCT from the nerve

tissue. Several nerves were mounted at each embryonic and neonatal age. Adult nerves were cut into short $(0.5-1 \mathrm{~cm})$ segments, before mounting. The unfixed nerves, mounted on liver, were rapidly frozen in isopentane precooled in liquid nitrogen. Tissue sections 6-9 $\mu \mathrm{m}$ thick were cut on a Bright cryostat, picked up on sterile polylysine-coated glass coverslips and stored at $-70^{\circ} \mathrm{C}$ in a sterile environment until required (Shewan et al., 1994). For each of the tissues tested as culture substrata for neurons, experiments were performed with at least five separate tissue preparations from different animals. 

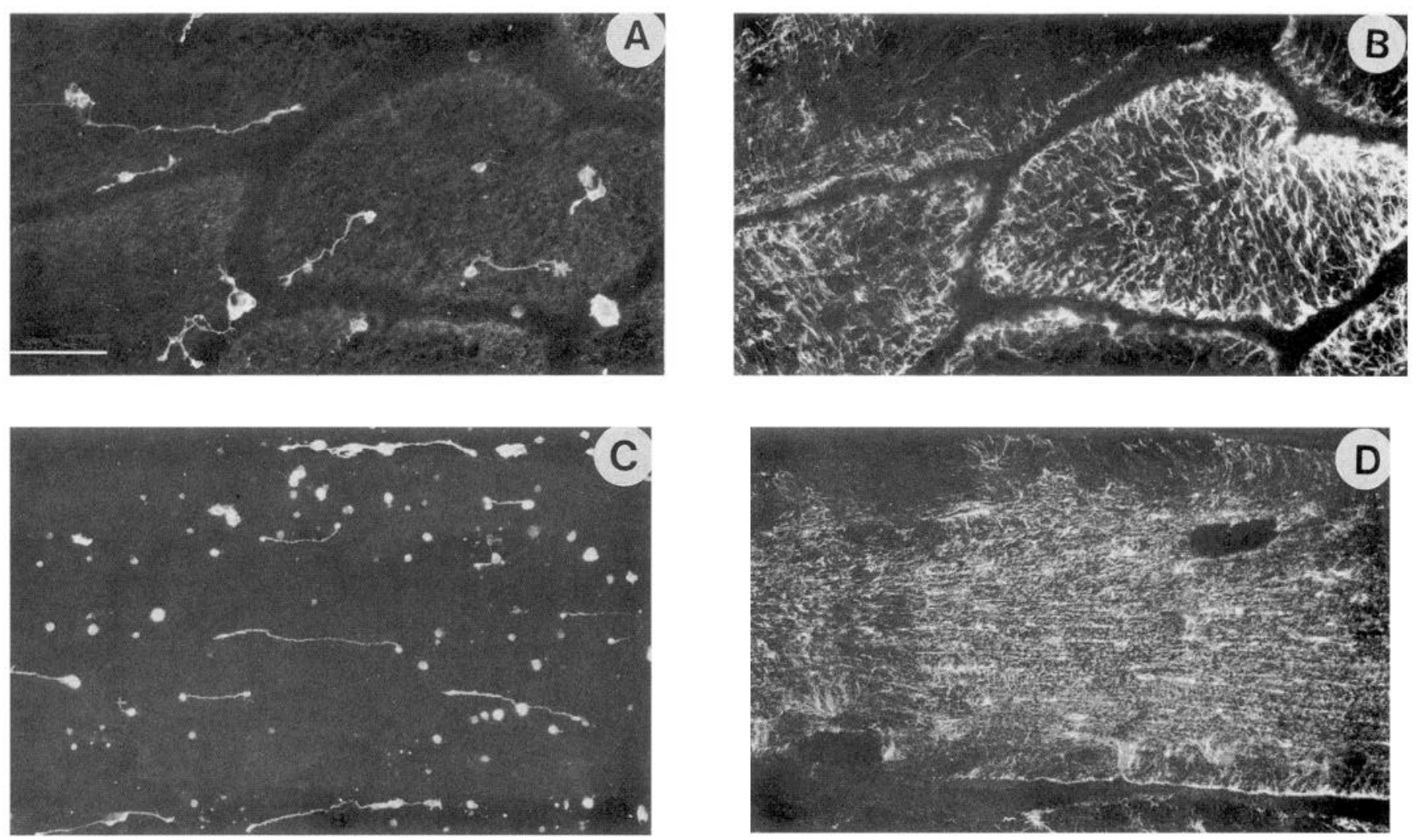

Figure 3. Fluorescence photomicrographs of double-immunolabeled cultures of early embryonic RGC on cryosections of neonatal $(A$ and $B$ ) and adult $(C$ and $D$ ) optic nerve tissue. E15 RGC regenerate most robustly on immature optic nerve sections $(A$ and $B)$ but can also extend long neurites on mature optic nerve cryosections $(C$ and $D)$. $A$ and $C$, GAP43; $B$ and $D$, GFAP. Scale bar $=100 \mu \mathrm{m}(A$ and $B)$ and $200 \mu \mathrm{m}(C$ and $D)$.

Preparation of dissociated retinal cells. Retinae from embryonic and perinatal (E14-P4) rats, were collected in calcium and magnesium-free Earles balanced salts solution (CMF; Gibco-BRL, UK), then transferred to $5 \mathrm{ml}$ papain (Lorne Laboratories, UK; $10 \mathrm{U} / \mathrm{ml}$ ) in a HEPES-buffered salt solution containing cysteine (Leifer et al., 1984), and incubated at $37^{\circ} \mathrm{C}$ for $15 \mathrm{~min}$. The tissue was then transferred to $0.5 \mathrm{ml} \mathrm{CMF/DNase}$ (Sigma UK, Type I; $50 \mu \mathrm{g} / \mathrm{ml}$ ) and triturated to give a single cell suspension by several passes through a small bore pipette tip. The mixed retinal cell suspension from E14-18 rats was plated at a concentration of 100,000 cells $/ \mathrm{ml}$.

Preparation of purified RGC. Purification of E20-P2 RGC from cell suspensions of neonatal rat retinae, by immunopanning for Thy-1-positive cells using monoclonal antibody (mab) Ox7, was achieved as described by Barres et al. (1988), except that the final step for removal of attached cells using trypsin was omitted; RGC were retrieved from the panning dish by vigorous pipetting with culture medium, and plated at a concentration of $60,000 / \mathrm{ml}$.

Preparation of dissociated DRG neurons. Sensory dorsal root ganglia were dissected out from embryonic, postnatal, and adult Wistar rats, collected in Ham's F-12 medium (Gibco, UK), and trimmed to remove attached roots. Embryonic and perinatal DRG tissue was enzymatically treated and cells dissociated as described for retinal tissue (see above). The dissociated cells were then diluted to a concentration of $5000 / \mathrm{ml}$ in modified Bottenstein and Sato's tissue culture medium containing $2 \%$ fetal calf serum (BSF2) in F-12, supplemented with nerve growth factor (NGF; $100 \mathrm{ng} / \mathrm{ml}$ ).

Adult ganglia were incubated at $37^{\circ} \mathrm{C}$ for $3 \mathrm{hr}$ in $0.125 \%$ collagenase (Sigma, UK), in F12, as described by Lindsay (1988), then transferred to papain solution and incubated for a further $15 \mathrm{~min}$, as described above, washed in F12, and triturated in fresh BSF2 $(1 \mathrm{ml})$. The cell suspension was spun through a $2 \mathrm{ml}$ cushion of $15 \%$ bovine serum albumin (fatty acid-free; Sigma, UK) in F-12, to remove myelin debris, and the pelleted cells resuspended in BSF2 containing NGF $(100 \mathrm{ng} / \mathrm{ml})$ to a cell concentration of $2000 / \mathrm{ml}$.

Neuronal cultures on tissue sections. The coverslips bearing cryostat sections were placed in plastic four-well multidishes (Nunc: Gibco, UK) and $0.5 \mathrm{ml}$ of cell suspensions were pipetted into each well. The dishes were placed in an incubator at $37^{\circ} \mathrm{C}$ with a humidified atmosphere of $5 \% \mathrm{CO}_{2}$ and $95 \%$ air. RGC and immature DRG neurons were cultured for a period of 18-24 hr, while adult DRG cells were incubated for 2$3 \mathrm{~d}$, prior to fixation and immunostaining.

Antibodies. Rabbit anti-GAP43 antiserum, from Rory Curtis and Graham Wilkin (Imperial College, London), was used as a marker for cultured neurons and their neurites at a dilution of 1:1000 (Bedi et al., 1992). Where mixed retinal cultures were employed, we confirmed by double immunolabeling that Thy-1-positive RGC were the only GAP43-positive cells present (data not shown). To visualize the underlying optic nerve tissue sections used as culture substrata, the antiGAP43 antiserum was combined in double immunostaining with a mouse monoclonal antibody to glial fibrillary acidic protein (GFAP; Sigma, UK), at a dilution of 1:400. In some function-blocking experiments, we used a rabbit anti- $\beta 1$ integrin antibody (as purified IgG, a gift of Kristofer Rubin, University of Uppsala, Sweden), diluted to a final concentration of $100 \mu \mathrm{g} / \mathrm{ml}$.

Immunohistochemistry. Cultures were fixed at room temperature for at least $10 \mathrm{~min}$ in $2 \%$ paraformaldehyde in phosphate-buffered saline (PBS) and permeabilized in $100 \%$ methanol at $-20^{\circ} \mathrm{C}$ for $3 \mathrm{~min}$. The coverslips were then washed three times in PBS and incubated at room temperature for $2 \mathrm{hr}$ (or overnight at $4^{\circ} \mathrm{C}$ ) in the diluted combinations of primary antibodies. All antibody dilutions were made in minimal Eagle's medium containing $10 \%$ fetal calf serum, 15 mM HEPES and $0.1 \%$ sodium azide. After further washes in PBS as above, the cultures were incubated for $30 \mathrm{~min}$ in a mixture of biotinylated anti-rabbit antibody (Amersham, UK) and fluorescein-conjugated anti-mouse antibody (Capel, USA), or a combination of biotinylated anti-mouse antibody (Amersham, UK) and fluorescein-conjugated anti-rabbit antibody (Sigma, UK), all diluted 1:100. After a further wash, the cultures were incubated for $30 \mathrm{~min}$ in Texas red-conjugated streptavidin (Amersham, UK), also at a dilution of 1:100. Following a final wash in PBS, the cultures were mounted in glycerol containing 1,4-diazobicyclo-(2,2,2) octane (DABCO; Sigma, UK) to prevent fading of the fluorescence. 

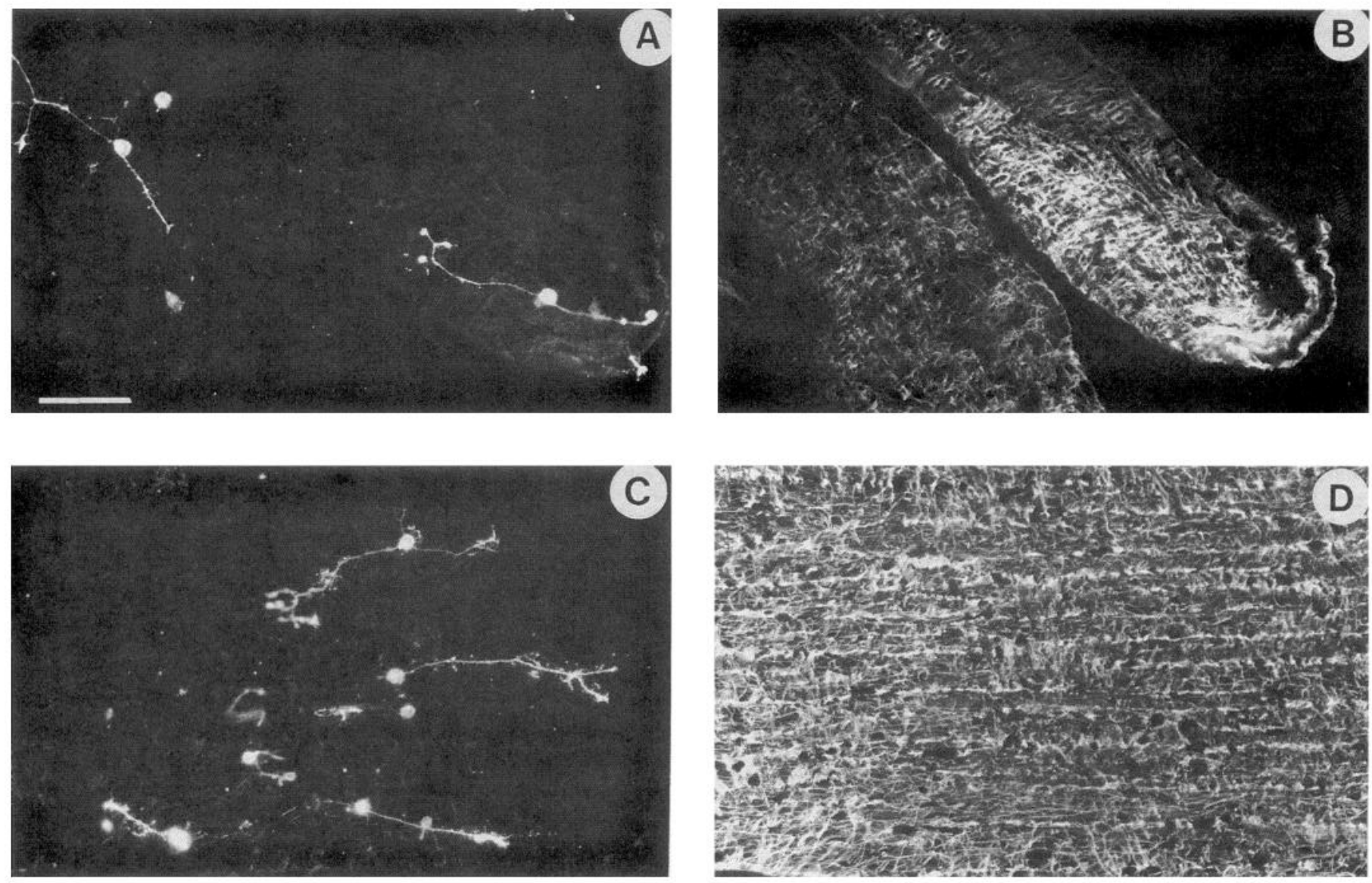

Figure 4. Fluorescence photomicrographs of double-immunolabeled cultures of early embryonic DRG neurons on cryosections of neonatal ( $A$ and $B)$ and adult $(C$ and $D)$ optic nerve tissue. E15 neurons regenerate extensively on immature optic nerve sections $(A$ and $B)$. Similarly, E15 DRG neurons grow prolifically on adult optic nerve tissue $(C$ and $D$ ). $A$ and $C$, GAP43; $B$ and $D$, GFAP. Scale bar $=100 \mu \mathrm{m}$.

The cultures were viewed with a Zeiss Axiophot microscope equipped with epifluorescence.

Cell counts. Counts were made under fluorescence optics of all GAP43-positive neurons attached to the tissue sections and of those with neurites of more than three cell body diameters (cbd) in length. The proportion of neurite-bearing cells was expressed as a percentage of the total number of attached cells in duplicate cultures, averaged for at least five separate preparations of each neuronal type \pm SEM, statistically evaluated by the Student's two tailed $t$ test. (Error bars that appear to be absent in Figures $1 B$ and $2 B$ are, in fact, too small to be plotted ( 0.3 and 0.4 , respectively.)

\section{Results}

We have previously shown that, in cryoculture, the failure of neonatal RGC and DRG neurons to extend neurites is unrelated to the myelination status of the CNS substrata (Shewan et al., 1993). When RGC from more immature embryonic rats are cultured on cryosections of unmyelinated, immature optic nerve, a substantial number extend processes, and this declines sharply with age (Fig. 1A). Thus, 19\% of E14-15 RGC that attached to unmyelinated, neonatal optic nerve sections extended long neurites ( $>3$ cbd), compared with only $4 \%$ of perinatal RGC, almost a fivefold reduction in responsive neurons. An example of this age dependence in neurite outgrowth is illustrated in Figure $3(A, B)$, where E15 RGC are seen to extend long GAP43-labeled processes over GFAP-positive neonatal unmyelinated optic nerve tissue sections. Although a similar trend was observed on myelinated adult optic nerve tissue sections (Fig. $1 B$; Fig. $3 C, D$ ), the magnitude of the response was reduced compared with the immature optic nerve.

In contrast to RGC, early embryonic DRG neurons grew equally well on both immature and myelinated optic nerve (Figs. $2,4)$. Moreover, this response declined sharply with age, with a comparable time course. Thus, almost half of the E14-15 DRG neurons that attached to optic nerve sections extended neurites, falling to less than $5 \%$ of neonatal DRG neurons (Fig. 2).

To test whether the reduction in outgrowth reflected a general decline over this period in the ability of DRG neurons to regenerate neurites, the behavior of cells that attached to section-free areas of polylysine-coated glass was also analyzed. Over the period E14-P4, the proportion of DRG neurons extending neurites on polylysine displayed only a modest reduction $(\sim 18 \%$; Fig. 2, inset). Also, $>80 \%$ of DRG neurons extended neurites when cultured on a laminin substratum, and this remained relatively constant over the same period (data not shown). Moreover, when cultured in the presence of blocking concentrations $(100 \mu \mathrm{g} / \mathrm{ml})$ of rabbit anti- $\beta 1$ integrin antibody, E14-15 DRG neurons displayed a somewhat reduced (approx. 30-35\% inhibited) neurite outgrowth response, over both myelinated adult and unmyelinated neonatal optic nerve (data not shown). These data suggest that integrin-binding ligand(s) in the optic nerve substratum play only a minor role in supporting the neurite outgrowth response of immature DRG neurons. 


\section{Discussion}

In this study we have examined the possibility that immature mammalian neurons are insensitive to putative growth inhibitory ligands in the CNS, by testing the ability of developing DRG neurons and RGC to regenerate neurites in vitro on both unmyelinated and myelinated optic nerve substrata. Our findings show that early embryonic DRG neurons will regenerate equally well on both types of optic nerve substrata, but this response declines sharply, and at a comparable rate on both substrata, by late embryonic age. This contrasts with the behavior of RGC, which exhibit a much reduced neurite outgrowth response, even at the earliest embryonic age, especially on myelinated optic nerve.

Concerning the nature of axon-growth inhibitors within the optic nerve, these findings extend our previous work (Shewan et al., 1993), and further implicate nonmyelin components as major CNS inhibitory ligands, recognized by late embryonic and mature neurons. Furthermore, the comparable magnitude of this inhibition on both types of optic ncrve, over an identical time course, is strongly indicative of a common inhibitory ligand(s), which seems more likely, therefore, to be located on optic nerve axons or astrocytes, rather than on oligodendrocytes. While Schwab and colleagues have identified proteins in CNS myelin that inhibit axon regeneration, and shown that antibodies raised against these molecules allow some regeneration in the injured CNS (Caroni and Schwab, 1988a,b; Schnell and Schwab, 1990), further evidence suggests the existence of other, astrocyte-derived, growth-inhibitory molecules, particularly since regeneration also fails in the injured unmyelinated grey matter of the CNS (Reier and Houle, 1988; Maxwell et al., 1990; Fawcett, 1992). Candidates include tenascin (Bartsch et al., 1992) and proteoglycans (Rudge and Silver, 1990; McKeon et al., 1991; Lander, 1993), the latter being able to repel neurites in vitro in the presence of outgrowth-promoting laminin (Snow et al., 1991). The expression of such molecules may contribute to the switch from a growth-permissive to an inhibitory environment during development, as suggested by Pindzola et al. (1993) for cytotactin and chondroitin 6-sulphate proteoglycans in the central portion of the dorsal root entry zone. Conversely, there is evidence that the expression of neurite growth-promoting molecules, such as laminin (Cohen et al., 1987) and heparan sulphate proteoglycan (Hantaz-Ambroise et al., 1987), are downregulated in the optic nerve in development (Liesi and Silver, 1988; McLoon et al., 1988; Ilalfter, 1993). Moreover, laminin-binding $\alpha 6 \beta 1$-integrin receptors are lost from RGC as they mature (Cohen et al., 1987, 1989; deCurtis et al., 1991), which may explain the loss of response to laminin, but not merosin, in development (Cohen et al., 1986; Cohen and Johnson, 1991). The demonstration in the present study of a marked agedependent decline in the ability of DRG neurons to regenerate in vitro on CNS tissue suggests that changes also occur in this class of mammalian neurons during embryonic development that influence their ability to regenerate within the CNS. These changes may not only involve the downregulation of receptors for growth promoting ligands, as in the case of RGC, but may also involve the acquisition of receptors for putative inhibitory CNS molecules.

An age-dependent decline in neurite outgrowth, most prominent on unmyelinated optic nerve, was also displayed by RGC, whose axons, unlike thuse of DRG neurons, project exclusively within the CNS. The fact that the earliest embryonic RGC tested did not display such a robust neurite outgrowth response on myelinated optic nerve suggests that they acquire receptors for these inhibitor(s) at an earlier age. This difference between the outgrowth responses of RGC and DRG neurons is not surprising, however, since distinct classes of neurons have previously been shown to have different axon-growth requirements. Thus, for example, neonatal RGC do not respond to the abundant growthpromoting molecules in cryosections of lesioned sciatic nerve that support DRG neurite outgrowth in vitro (Shewan et al., 1993). Moreover, axons regenerating from motor and sensory neurons appear to respond differentially to molecules expressed in their peripheral distal pathways (Martini et al., 1992).

Our in vitro data, and the in vivo transplantation studies of Wictorin et al. (1990, 1992), are consistent with the hypothesis that early embryonic neurons that are actively extending axons in vivo do not recognize molecules within the CNS that block axon regeneration by older neurons. The timing of this change suggests that the acquisition by neurons of receptors for such molecules, and the progressive restriction of growth by regenerating axons in the injured CNS, may occur only after these axons contact their normal targets in vivo (Fitzgerald and Fulton, 1992). The nature of these receptors remain to be determined, but the findings of the present study may offer an experimental approach by directing attention to differences between early embryonic and older neurons in the repertoire of membrane receptors for identified axon-growth inhibitory molecules.

\section{References}

Barres BA, Silverstein BE, Corey DP, Chun LL (1988) Immunological, morphological and electrophysiological variation among retinal cells purified by panning. Neuron 1:791-803.

Bartsch U, Bartsch S, Dorries U, Schachner M (1992) Immunohistological localization of tenascin in the developing and lesioned adult mouse optic nerve. Eur J Neurosci 4:338-352.

Bedi KS, Winter J, Berry M, Cohen J (1992) Adult rat dorsal root ganglion neurons extend neurites on predegenerated but not on normal peripheral nerves in vitro. Eur J Neurosci 4:193-200.

Benfey M, Aguayo AJ (1982) Extensive elongation of axons from rat brain into peripheral nerve grafts. Nature 296:150-152.

Carbonetto S, Evans D, Cochard P (1987) Nerve fiber growth in culture on tissue substrates from central and peripheral nervous system. J Neurosci 7:610-620.

Caroni P, Schwab ME (1988a) Two membrane protein fractions from rat central myelin with inhibitory properties for neurite growth and fibroblast spreading. J Cell Biol 106:1281-1288.

Caroni P, Schwab ME (1988b) Antibody against myelin-associated inhibitors of neurite growth neutralizes nonpermissive substrate properties of CNS white matter. Neuron 1:85-96.

Cohen J, Johnson AR (1991) Differential effects of laminin and merosin on neuritc outgrowth by developing retinal ganglion cclls. J Ccll Sci (Suppl.) 15:1-7.

Cohen J, Burne JF, Winter J, Bartlett PF (1986) Retinal ganglion cells lose response to laminin with maturation. Nature 322:465-467.

Cohen J, Burne JF, McKinley C, Winter J (1987) The role of laminin and the laminin/fibronectin receptor complex in the outgrowth of retinal ganglion cell axons. Dev Biol 122:407-418.

Cohen J, Nurcombe V, Jeffrey P, Edgar D (1989) Developmental loss of functional laminin receptors on retinal ganglion cells is regulated by their target tissue the optic tectum. Development 107:381-387.

Covault J, Cunningham JM, Sanes JR (1987) Neurite outgrowth on cryosections of innervated and denervated skeletal muscle. J Cell Biol 105:2479-2488.

David S, Aguayo AJ (1981) Axonal elongation into peripheral nervous system "bridges" after central nervous system injury in adult rats. Science 214:931-933.

deCurtis I, Quaranta V, Tamura RN, Reichardt LF (1991) Laminin receptors in the retina: sequence analysis of the chick integrin $\alpha 6$ subunit. J Cell Biol 113:405-416. 
Fawcett JW (1992) Intrinsic neuronal determinants of regeneration. Trends Neurosci 15:5-8.

Fawcett JW, Housden E, Smith-Thomas L, Meyer RL (1989) The growth of axons in three-dimensional astrocyte cultures. Dev Biol 135:449-458.

Fitzgerald M, Fulton BP (1992) The physiological properties of developing sensory neurons. In: Sensory neurons: diversity, development and plasticity (Scott S, ed), pp 287-306. New York: Oxford UP.

Halfter W (1993) A heparan sulfate proteoglycan in developing avian axonal tracts. J Neurosci 13:2863-2873.

Hantaz-Ambroise D, Vigny M, Koenig J (1987) Heparan sulfate proteoglycan and laminin mediate two different types of neurite outgrowth. J Neurosci 7:2293-2304.

Johnson AR (1994) Contact inhibition in the failure of mammalian CNS axonal regeneration. Bioessays 15:807-813.

Lander AD (1993) Proteoglycans in the nervous system. Curr Opin Neurobiol 3:716-723.

Leifer D, Lipton SA, Barnstaple CJ, Masland SH (1984) Monoclonal antibody to Thy-1 enhances regeneration of processes by rat retinal ganglion cells in culture. Science 232:303-306.

Liesi P, Silver J (1988) Is astrocyte laminin involved in axon guidance in the mammalian CNS? Dev Biol 130:774-785.

Lindsay RM (1988) Nerve growth factors (NGF, BDNF) enhance axonal regeneration but are not required for survival of adult sensory neurons. J Neurosci 8:2394-2405.

McKeon RJ, Schreiber RC, Rudge JS, Silver J (1991) Reduction of neurite outgrowth in a model of glial scarring CNS injury is correlated with the expression of inhibitory molecules on reactive astrocytes. J Neurosci 11:3398-3411.

McLoon SC, McLoon LK, Palm SL, Furcht LT (1988) Transient expression of laminin in the optic nerve of the developing rat. $J$ Neurosci 8:1981-1990.

Martini R, Xin Y, Schmitz B, Schachner M (1992) The L2/HNK-1 carbohydrate epitope is involved in the preferential outgrowth of mo- tor neurons on ventral roots and motor nerves. Eur J Neurosci 4:628639.

Maxwell WL, Follows R, Ashurst E, Berry M (1990) The response of the cerebral hemispheres of the rat to injury. I. The mature rat. Philos Trans R Soc Lond [Biol] 328:479-500.

Pindzola RR, Doller C, Silver J (1993) Putative inhibitory extracellular matrix molecules at the dorsal root entry zone of the spinal cord during development and after root and sciatic nerve lesions. Dev Biol 156:34-48.

Reier PJ, Houle JD (1988) The glial scar: its bearing on axonal elongation and transplantation approaches to CNS repair. In: Advances in neurology: functional recovery in neurological disease (Waxman SG, ed), pp 87-138. New York: Raven.

Rudge JS, Silver J (1990) Inhibition of neurite outgrowth on astroglial scars in vitro. J Neurosci 10:3594-3603.

Sandrock AW, Matthew WD (1987) Identification of a peripheral nerve neurite-outgrowth promoting activity by development and use of an in vitro bioassay. Proc Natl Acad Sci USA 84:6934-6938.

Schnell I, Schwab ME (1990) Axonal regeneration in the rat spinal cord produced by an antibody against myelin-associated neurite growth inhibitors. Nature 343:269-272.

Shewan D, Berry M, Bedi K, Cohen J (1993) Embryonic optic nerve tissue fails to support neurite outgrowth by central and peripheral neurons in vitro. Eur J Neurosci 5:809-817.

Shewan D, Bedi K, Berry M, Winter J, Cohen J (1994) Axon regeneration in vitro on physiologically relevant substrata. Neuroprotocols $4: 142-145$.

Snow DM, Watanabe M, Letourneau PC, Silver J (1991) A chondroitin sulfate proteoglycan may influence the direction of retinal ganglion cell outgrowth. Development 113:1473-1486.

Wictorin K, Brundin P, Gustavii B, Lindvall O, Björklund A (1990) Reformation of long axon pathways in adult rat central nervous system by human forebrain neuroblasts. Nature 347:556-558

Wictorin K, Brundin P, Sauer H, Lindvall O, Björklund A (1992) Long distance directed axonal growth from human dopaminergic mesencephalic neuroblasts implanted along the nigrostriatal pathway in 6-hydroxydopamine lesioned adult rats. J Comp Neurol 323:475-494. 